\title{
CUSTOMS MUTUAL ASSISTANCE AGREEMENTS IN THE LIGHT OF ARTICLE 12 TFA - A GLOBAL PERSPECTIVE
}

Customs co-operation at international, regional and global levels is aimed at ensuring compliance with applicable laws and regulations and improving trade flow control through the exchange of information on Customs aspects such as transit, export and import declaration data, origin and valuation-related information, trader-related information and in particular information on customs fraud. This exchange of data is currently a key element of the WTO TFA and Article 12 of the TFA considers all the necessary components for such an exchange of information, such as the requesting country's need, verify its request, protect the exchanged data, and ensure the confidentiality of data and the exchange of data based on the principle of reciprocity. Article 12 TFA also refers to bilateral and regional mutual administrative assistance agreements, which remain the main tool for governments and customs administrations to engage in such cooperation. This paper investigates the different approaches and lists the Customs Mutual Assistance Agreements (CMAAs) of mayor stakeholders in Global Trade, of medium trade nations and of small countries. 23 tables are displaying the results of this research. It investigates CMAAs by help of direct publications on their CMAAs and of other nations that are not publishing data on their CMAAs by pooling data published by other nations. The paper concludes that bilateral mutual agreements are favorable for large nations but difficult to negotiate for small nations. Finally it calls for the importance of regional mutual customs cooperation.

Key words: Customs law, Customs classification, Tariff schedule, Harmonized System (HS) Nomenclature, General Rules, Headings, Notes, Subheading notes, Additional notes, Common Customs Tariff (CCT), Combined Nomenclature (CN), European Union (EU).

JEL Classification: F 15, F 53, K 42.

\section{Carsten WEERTH,}

Main Customs Office Bremen,

Federal Customs Service of Germany, FOM University of Applied Sciences in Economics and Management, Bremen, Germany carsten.weerth@gmx.de

\section{Introduction}

Simplification of trade and transport procedures is interpreted in a broad sense, as the simplification and harmonization of procedures and documentation for international trade and transport. It creates a favorable business environment, reduces the overall cost of international trade and transport, applying best international practices in national procedures. The multilateral trade negotiations under the auspices of the World Trade Organization (WTO) have significantly reduced tariff barriers. This paper introduces the different tools for customs cooperation and it investigates for the first time the number of Customs Mutual Assistance Agreements (CMAAs) of major World Trade Nations (EU, US, Australia, Canada, China, Japan, New Zealand, South Africa and South Korea), of medium trade nations (India, Turkey and UAE) also of small countries (Algeria, Mauritius, Morocco, Hong Kong and Taiwan). It investigates CMAAs by help of direct publications on their CMAAs and of other nations that are not publishing data on their CMAAs by pooling data published by other nations. 


\subsection{Legal Framework for Customs cooperation}

The World Customs Organization (WCO) has developed a framework for Customs cooperation which consists of different binding and non-binding instruments.

There are various obligatory and optional legal instruments provided by international organizations - the WCO and other international economic organizations such as the United Nations: ${ }^{1}$

WCO-Recommendations (non-binding)

- 1953 Council Recommendation on Mutual Administrative Assistance

- 1967 Recommendation of the Customs Co-operation Council on the Pooling of Information concerning Customs Fraud

- 1975 Council Recommendation on the Pooling of Information concerning Customs Fraud

WCO-Declarations (non-binding)

- 2000 Cyprus Declaration

WCO: Binding Instruments

- Revised Model Bilateral Agreement - June 2004

- Guides for Regional Mutual Administrative Assistance in Customs matters (2002)

- Convention on mutual assistance and cooperation between customs administrations (Naples II)

- Council Regulation on Mutual Assistance for Application of the Law on Customs (1997)

- Convention on the Use of Information Technology for Customs Purposes (1995)

- International Convention on mutual administrative assistance for the prevention, investigation and repression of Customs offences (Nairobi Convention, JC) (1980)

- International Convention on mutual administrative assistance in Customs matters (Johannesburg Convention) (has not entered into force)

- United Nations Instruments (binding)

- Convention Against Illicit Traffic in Narcotic Drugs and Psychotropic Substances, 1998 (Vienna Convention)

- Convention Against Transnational Organized Crime, 2000 (Palermo Convention)

- UNCITRAL Model Law on Electronic Commerce with Guide to Enactment, 1996

What one can state after this long list of legal instruments is that mutual customs assistance is done on the basis of the NC but not only and in many cases with other WCO and/or UN instruments. In particular bilateral customs mutual assistance agreements (CMAAs) have been drawn by help of the WCO since its first publication in 1967.

\subsection{Article 12 TFA}

Article 12 of the Trade Facilitation Agreement (TFA) ${ }^{2}$ is concerned with customs cooperation. This topic is of eminent importance for Trade Facilitation because risk analysis and mutual data exchange is part of the global trade picture.

In particular, Art. 12 TFA provides a list of terms and requirements for the Member States concerning the exchange of information. It is intended to monitor confidentiality when exchanging information. This article allows member states of the WTO within the framework of national legal systems to set a timeframe for the provision and receipt of information. Moreover, WTO member states can even conclude or maintain bilateral, multilateral or regional agreements for the exchange of customs information. Sections 1, 3, 6 and 7 of the RKC contain provisions for cooperation between customs administrations. For example, Standard 6.7 provides the following: "Customs should seek cooperation with other customs administrations and conclude agreements on mutual administrative assistance with a view to improving customs control".

As for the use of information technology, the more specific provisions on this are contained in the RKC. Chapter 7 of this Convention provides for the use of information technology by the customs authorities in cases where it is economically profitable and effective for the customs service and participants in trade activities.

\footnotetext{
${ }^{1}$ See WCO, Mutual Administrative Assistance (2018b), URL: http://www.wcoomd.org/en/topics/enforcement-and-compliance/instruments-and-tools/wco-and-international-instruments-on-mutual-administrativeassistance.aspx, viewed 8 April 2018.
}

${ }^{2}$ The full text is available in English under URL: https://www.wto.org/english/docs_e/legal_e/tfa-nov14_e.htm. 
It should be noted that when implementing information technology, consultations are conducted with all parties that have a direct relation to it $(7.3, \mathrm{RKC})$. Also, in Standard 7.4 of the $\mathrm{RKC}$ it is envisaged that new or amended legislation of the states that have ratified the provisions of the RKC should provide for: 1) electronic methods for the exchange of commercial information; 2) the combination of electronic methods and methods for certifying the authenticity and identity of documents on paper; 3 ) the right of the customs service to keep information for use for customs purposes and, if necessary, exchange such information with other customs administrations, as well as legal entities

\section{Examination of the Customs Mutual Assistance Agreements (MCAAs)}

\subsection{Material and Methods}

This examination was conducted by help of an internet research regarding data on CMAAs of major global trade nations and also of medium and minor trade nations which have been published by them. Furthermore data has been obtained about other parties which have not published any data on their CMAAs by help data pooling of other publications.

\subsection{Results}

The results of direct publications are shown in Tables 1-14.

Customs Mutual Assistance Agreements (CMAAs) of the US

Table 1

\begin{tabular}{|c|c|c|}
\hline Country & WCO & WTO \\
\hline Algeria & + & $-*$ \\
\hline Argentina & + & + \\
\hline Australia & + & + \\
\hline Austria & + & + \\
\hline Azerbaijan & + & $-*$ \\
\hline Bahrain & + & + \\
\hline Belarus & + & $-*$ \\
\hline Belgium & + & + \\
\hline Brazil & + & + \\
\hline Bulgaria & + & + \\
\hline Canada & + & + \\
\hline Chile & + & + \\
\hline China & + & + \\
\hline Colombia & + & + \\
\hline Costa Rica & + & + \\
\hline Cyprus & + & + \\
\hline Czech Republic & + & + \\
\hline Denmark & + & + \\
\hline Dominican Republic & + & + \\
\hline Ecuador & + & + \\
\hline European Community & + & + \\
\hline Finland & + & + \\
\hline France & + & + \\
\hline Gabon & + & + \\
\hline Germany & + & + \\
\hline
\end{tabular}

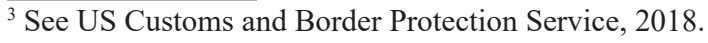




\begin{tabular}{|c|c|c|}
\hline Greece & + & + \\
\hline Honduras & + & + \\
\hline Hong Kong & + & + \\
\hline Hungary & + & + \\
\hline India & + & + \\
\hline Indonesia & + & + \\
\hline Ireland & + & + \\
\hline Israel & + & + \\
\hline Italy & + & + \\
\hline Japan & + & + \\
\hline Jordan & + & + \\
\hline Kasakhstan & + & + \\
\hline Kenya & + & + \\
\hline Latvia & + & + \\
\hline Lithuania & + & + \\
\hline Malaysia & + & + \\
\hline Maldives & + & + \\
\hline Malta & + & + \\
\hline Mauritius & + & + \\
\hline Mexico & + & + \\
\hline Mongolia & + & + \\
\hline Montenegro & + & + \\
\hline Morocco & + & + \\
\hline Netherlands & + & + \\
\hline New Zealand & + & + \\
\hline Nigeria & + & + \\
\hline Norway & + & + \\
\hline Pakistan & + & + \\
\hline Panama & + & + \\
\hline Peru & + & + \\
\hline Philippines & + & + \\
\hline Poland & + & + \\
\hline Portugal & + & + \\
\hline Romania & + & + \\
\hline Russian Federation & + & + \\
\hline Senegal & + & + \\
\hline Serbia & + & $-*$ \\
\hline Singapore & + & + \\
\hline Slovakia & + & + \\
\hline South Africa & + & + \\
\hline South Korea & + & + \\
\hline Spain & + & + \\
\hline Sweden & + & + \\
\hline
\end{tabular}




\begin{tabular}{|c|c|c|}
\hline Taiwan & - & + \\
\hline Trinidad \& Tobago & + & + \\
\hline Turkey & + & + \\
\hline Ukraine & + & + \\
\hline United Kingdom & + & + \\
\hline Uruguay & + & + \\
\hline Venezuela & + & + \\
\hline Summary 75 CMAAs & 74 & 71 \\
\hline
\end{tabular}

*WTO Observer

Table 2

Customs Mutual Assistance Agreements (CMAAs) of Canada ${ }^{4}$

\begin{tabular}{|c|c|c|}
\hline Country & WCO & WTO \\
\hline European Community & + & + \\
\hline France & + & + \\
\hline Germany & + & + \\
\hline Mexico & + & + \\
\hline Netherlands & + & + \\
\hline South Africa & + & + \\
\hline South Korea & + & + \\
\hline USA & + & + \\
\hline Summary 8 CMAAs & 8 & 8 \\
\hline
\end{tabular}

Table 3

Customs Information Sharing Memorandum of Understandings (MOUs) of Canada

\begin{tabular}{|c|c|c|}
\hline Country & WCO & WTO \\
\hline Australia & + & + \\
\hline Caribbean Zone & + & - \\
\hline Great Britain (UK) & + & + \\
\hline Mexico & + & + \\
\hline Hong Kong & + & + \\
\hline Japan & + & + \\
\hline New Zealand & + & + \\
\hline Summary 7 MOUs & 7 & 6 \\
\hline
\end{tabular}

*WTO Observer

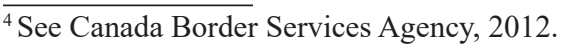

${ }^{5}$ See Canada Border Services Agency, 2012.

Customs Scientific Journal, № 1, 2019 
Table 4

Customs Information Sharing Mutual Legal Assistance Treaties (MLTAs) of Canada ${ }^{6}$

\begin{tabular}{|c|c|c|}
\hline Country & WCO & WTO \\
\hline Argentina & + & + \\
\hline Austria & + & + \\
\hline Australia & + & + \\
\hline Bahamas & + & $-*$ \\
\hline Belgium & + & + \\
\hline Brazil & + & + \\
\hline China & + & + \\
\hline Czech Republic & + & + \\
\hline France & + & + \\
\hline Germany & + & + \\
\hline Greece & + & + \\
\hline Hong Kong & + & + \\
\hline Hungary & + & + \\
\hline India & + & + \\
\hline Israel & + & + \\
\hline Italy & + & + \\
\hline Mexico & + & + \\
\hline Netherlands & + & + \\
\hline Norway & + & + \\
\hline Peru & + & + \\
\hline Poland & + & + \\
\hline Portugal & + & + \\
\hline Romania & + & + \\
\hline Russian Federation & + & + \\
\hline South Africa & + & + \\
\hline South Korea & + & + \\
\hline Spain & + & + \\
\hline Switzerland & + & + \\
\hline Sweden & + & + \\
\hline Thailand & + & + \\
\hline Trinidad and Tobago & + & + \\
\hline Ukraine & + & + \\
\hline United Kingdom & + & + \\
\hline Uruguay & + & + \\
\hline USA & + & + \\
\hline Summary 35 MLTAs & 35 & 34 \\
\hline
\end{tabular}

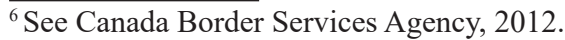


Customs Mutual Assistance Agreements (CMAA) of Taiwan?

Table 5

\begin{tabular}{|c|c|c|}
\hline Country & WCO & WTO \\
\hline Canada & + & + \\
\hline China & + & + \\
\hline India & + & + \\
\hline Israel & + & + \\
\hline Macedonia & + & - \\
\hline New Zealand & + & + \\
\hline Philippines & + & + \\
\hline United States & + & + \\
\hline Summary 8 CMAAs & 8 & 7 \\
\hline
\end{tabular}

Customs Mutual Assistance Agreements (CMAAs) of Japan ${ }^{8}$

Table 6

\begin{tabular}{|c|c|c|}
\hline Country & WCO & WTO \\
\hline China & + & + \\
\hline European Community & + & + \\
\hline Germany & + & + \\
\hline Italy & + & + \\
\hline Mexico & + & + \\
\hline Netherlands & + & + \\
\hline Norway & + & + \\
\hline Russian Federation & + & + \\
\hline South Africa & + & + \\
\hline South Korea & + & + \\
\hline Spain & + & + \\
\hline USA & + & + \\
\hline Summary 12 CMAAs & 12 & 12 \\
\hline
\end{tabular}

*WTO Observer

Table 7

Customs Mutual Assistance Arrangements of Japan'

\begin{tabular}{|c|c|c|}
\hline Country & WCO & WTO \\
\hline Australia & + & + \\
\hline Belgium & + & + \\
\hline Canada & + & + \\
\hline France & + & + \\
\hline Hong Kong & + & + \\
\hline Macao & - & + \\
\hline New Zealand & + & + \\
\hline United Kingdom & + & 8 \\
\hline Summary 7 CMAAs & 7 & \\
\hline
\end{tabular}

${ }^{7}$ See Republic of China (Taiwan), 2018.

${ }^{8}$ See Japan Customs, 2018.

${ }^{9}$ See Japan Customs, 2018.

Customs Scientific Journal, № 1, 2019 
Table 8

Customs Mutual Assistance Agreements (CMAA) of the European Union (EU) ${ }^{10}$

\begin{tabular}{|c|c|c|}
\hline Country & WCO & WTO \\
\hline Albania & + & + \\
\hline Algeria & + & $-*$ \\
\hline Andorra & + & $-*$ \\
\hline Antigua and Barbuda & + & + \\
\hline Armenia & + & + \\
\hline Azerbaijan & + & $-*$ \\
\hline Bahamas & + & $-*$ \\
\hline Barbados & + & + \\
\hline Belize & + & + \\
\hline Bosnia and Herzegovina & + & $-*$ \\
\hline Botswana & + & + \\
\hline Cameroon & + & + \\
\hline Canada & + & + \\
\hline Chile & + & + \\
\hline China & + & + \\
\hline Colombia & + & + \\
\hline Costa Rica & + & + \\
\hline Dominica & - & + \\
\hline Dominican Republic & + & + \\
\hline Egypt & + & + \\
\hline Faroe Islands & - & - \\
\hline Fiji & + & + \\
\hline Georgia & + & + \\
\hline Ghana & + & + \\
\hline Grenada & - & + \\
\hline Guatemala & + & + \\
\hline Guyana & + & + \\
\hline Honduras & + & + \\
\hline Hong Kong & + & + \\
\hline Iceland & + & + \\
\hline Ivory Coast & + & + \\
\hline India & + & + \\
\hline Israel & + & + \\
\hline Jamaica & + & + \\
\hline Japan & + & + \\
\hline Jordan & + & + \\
\hline Kazakhstan & + & + \\
\hline Kosovo & + & - \\
\hline Kyrgyzstan & + & + \\
\hline Lebanon & + & $-*$ \\
\hline Lesotho & + & + \\
\hline Liechtenstein & - & + \\
\hline Macedonia/FYROM & + & - \\
\hline Madagascar & + & + \\
\hline Mauritius & + & + \\
\hline Mexico & + & + \\
\hline
\end{tabular}

${ }^{10}$ See European Commission, OLAF, Legal Framework, Agreement with third parties, 2017 and European Commission, Press Releases on the EU-New Zealand CMAA, 2017. 


\begin{tabular}{|c|c|c|}
\hline Moldova & + & + \\
\hline Montenegro & + & + \\
\hline Morocco & + & + \\
\hline Mozambique & + & + \\
\hline Namibia & + & + \\
\hline New Zealand & + & + \\
\hline Nicaragua & + & + \\
\hline Norway & + & + \\
\hline Palestinian Authority & + & - \\
\hline Panama & + & + \\
\hline Papua New Guinea & + & + \\
\hline Peru & + & + \\
\hline Russian Federation & + & + \\
\hline Saint Christopher and Nevis & - & + \\
\hline Saint Lucia & + & + \\
\hline $\begin{array}{c}\text { Saint Vincent and the } \\
\text { Grenadines }\end{array}$ & - & + \\
\hline El Salvador & + & + \\
\hline San Marino & - & - \\
\hline Serbia & + & - \\
\hline Seychelles & + & + \\
\hline South Africa & + & + \\
\hline South Korea & + & + \\
\hline Suriname & - & + \\
\hline Swaziland & + & + \\
\hline Switzerland & + & + \\
\hline Tajikistan & + & + \\
\hline Trinidad and Tobago & + & + \\
\hline Tunisia & + & + \\
\hline Turkey & + & + \\
\hline Turkmenistan & + & - \\
\hline Ukraine & + & + \\
\hline USA & + & + \\
\hline Uzbekistan & + & $-*$ \\
\hline Zimbabwe & + & + \\
\hline Summary 80 CMAAs & 72 & 66 \\
\hline
\end{tabular}

*WTO Observer 
Customs Mutual Assistance Agreements (CMAAs) of India ${ }^{11}$

Table 9

\begin{tabular}{|c|c|c|}
\hline Country & WCO & WTO \\
\hline Australia & + & + \\
\hline Brazil & + & + \\
\hline China & + & + \\
\hline Egypt & + & + \\
\hline European Union & + & + \\
\hline Hong Kong & + & + \\
\hline Iran & + & $-*$ \\
\hline Israel & + & + \\
\hline Maldives & + & + \\
\hline Russian Federation & + & + \\
\hline SAARC & - & - \\
\hline South Korea & + & + \\
\hline United Kingdom & + & + \\
\hline USA & + & + \\
\hline Uzbekistan & + & $-*$ \\
\hline Summary 15 CMAAs & 14 & 12 \\
\hline
\end{tabular}

*WTO Observer

Table 10

Customs Mutual Assistance Agreements (CMAAs) of the United Arab Emirates ${ }^{12}$

\begin{tabular}{|c|c|c|}
\hline Country & WCO & WTO \\
\hline Algeria & + & $-*$ \\
\hline Argentina & + & + \\
\hline Armenia & + & $-^{*}$ \\
\hline Azerbeijan & + & + \\
\hline India & + & + \\
\hline Kazakhstan & + & + \\
\hline Maldives & + & + \\
\hline Morocco & + & + \\
\hline Netherlands & + & + \\
\hline Pakistan & + & 9 \\
\hline South Korea & + & + \\
\hline Summary 11 CMAAs & 11 & + \\
\hline
\end{tabular}

*WTO Observer

Table 11

Customs Mutual Assistance Agreements (CMAAs) of Mauritius ${ }^{13}$

\begin{tabular}{|c|c|c|}
\hline Country & WCO & WTO \\
\hline Netherlands & + & + \\
\hline Pakistan & + & + \\
\hline USA & + & + \\
\hline Summary 3 CMAAs & 3 & 3 \\
\hline
\end{tabular}

${ }^{11}$ See Government of India, 2018.

${ }^{12}$ See UAE Customs Service, 2018.

${ }^{13}$ See Mauritian Customs Service, 2018. 
Customs Mutual Assistance Agreements (CMAAs) of Turkey which have entered into force ${ }^{14}$

\begin{tabular}{|c|c|c|}
\hline Country & WCO & WTO \\
\hline Afghanistan & + & + \\
\hline Albania & + & + \\
\hline Algeria & + & $-*$ \\
\hline Bahrain & + & + \\
\hline Belarus & + & $-*$ \\
\hline Belgium & + & + \\
\hline Bosnia-Herzegovina & + & $-*$ \\
\hline Bulgaria & + & + \\
\hline Chile & + & + \\
\hline China & + & + \\
\hline Croatia & + & + \\
\hline Cuba & + & + \\
\hline Czech Republic & + & + \\
\hline D-8 & - & + \\
\hline Egypt & + & + \\
\hline Estonia & + & + \\
\hline Georgia & + & + \\
\hline Greece & + & + \\
\hline India & + & + \\
\hline Iran & + & $-*$ \\
\hline Israel & + & + \\
\hline Italy & + & + \\
\hline Jordan & + & + \\
\hline Kazakhstan & + & + \\
\hline Kosovo & + & - \\
\hline Kuwait & + & + \\
\hline Kyrgyzstan & + & + \\
\hline Latvia & + & + \\
\hline Lithuania & + & + \\
\hline Macedonia & + & - \\
\hline Mexico & + & + \\
\hline Moldova & + & + \\
\hline Mongolia & + & + \\
\hline Morocco & + & + \\
\hline Netherlands & + & + \\
\hline Pakistan & + & + \\
\hline Palestine & + & - \\
\hline Poland & + & + \\
\hline Romania & + & + \\
\hline Russian Federation & + & + \\
\hline Saudi Arabia & + & + \\
\hline Serbia & + & $-*$ \\
\hline Slovakia & + & + \\
\hline Slovenia & + & + \\
\hline South Africa & + & + \\
\hline South Korea & + & + \\
\hline
\end{tabular}

${ }^{14}$ See Turkish Customs Service, 2018.

Customs Scientific Journal, № 1, 2019 


\begin{tabular}{|c|c|c|}
\hline Spain & + & + \\
\hline Sudan & + & $-*$ \\
\hline Syria & + & $-*$ \\
\hline Tajikistan & + & + \\
\hline Turkmenistan & + & - \\
\hline Ukraine & + & + \\
\hline United Kingdom & + & + \\
\hline USA & + & + \\
\hline Uzbekistan & + & $-*$ \\
\hline Summary 55 CMAAs & 54 & 43 \\
\hline
\end{tabular}

*WTO Observer

Table 13

Customs Mutual Assistance Agreements (CMAAs) of Turkey which have not entered into force ${ }^{15}$

\begin{tabular}{|c|c|c|}
\hline Country & WCO & WTO \\
\hline Azerbaijan & + & $-^{*}$ \\
\hline Bangladesh & + & + \\
\hline Brazil & + & + \\
\hline Libya & + & $+*$ \\
\hline Qatar & + & + \\
\hline Montenegro & + & + \\
\hline Oman & + & + \\
\hline Tunisia & + & + \\
\hline Yemen & + & 7 \\
\hline Summary 9 CMAAs & 9 & + \\
\hline
\end{tabular}

*WTO Observer

Table 14

Customs Mutual Assistance Agreements (CMAA) of Argentina ${ }^{16}$

\begin{tabular}{|c|c|c|}
\hline Country & WCO & WTO \\
\hline Azerbeijan & + & + \\
\hline Bolivia & + & + \\
\hline Chile & + & + \\
\hline France & + & + \\
\hline Hungary & + & + \\
\hline India & + & + \\
\hline Italy** & + & + \\
\hline Libya & + & $-*$ \\
\hline MERCOSUR & - & - \\
\hline Russian Federation & + & + \\
\hline Spain & + & + \\
\hline USA & + & + \\
\hline Summary 12 CMAAs & 11 & 10 \\
\hline
\end{tabular}

*WTO Observer

**Agreement pending parliamentarian approval

${ }^{15}$ See Turkish Customs Service, 2018.

${ }^{16}$ See Argentinean Customs Service, 2018. 
14 tables of direct publications by countries are showing the different approaches of the countries investigated which have published data on mutual customs assistance agreements. This study is incomplete (since it only covers only about 10 out of 164 WTO and 182 WCO member states) but it gives a deep insight in this secretive topic of customs assistance.

Some data on CMAAs can be obtained by help of data pooling by publications about some other countries such as Algeria, Argentina, China, Hong Kong, Morocco, Russia, Australia, New Zealand, South Africa and South Korea (tables 15-23).

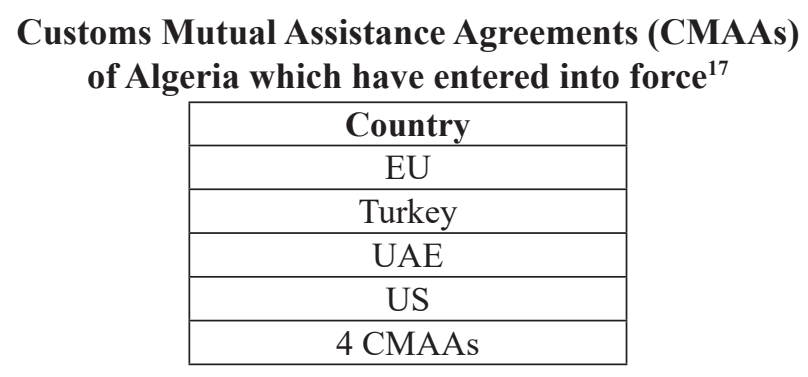

Table 15

Customs Mutual Assistance Agreements (CMAAs)

Table 16 of China which have entered into force ${ }^{18}$

\begin{tabular}{|c|}
\hline Country \\
\hline Canada \\
\hline EU \\
\hline India \\
\hline Japan \\
\hline Taiwan \\
\hline Turkey \\
\hline US \\
\hline 7 CMAAs \\
\hline
\end{tabular}

Customs Mutual Assistance Agreements (CMAAs) of Hong Kong which have entered into force ${ }^{19}$

\begin{tabular}{|c|}
\hline Country \\
\hline Canada \\
\hline EU \\
\hline India \\
\hline Japan \\
\hline US \\
\hline 7 CMAAs \\
\hline
\end{tabular}

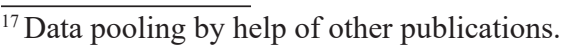

${ }^{18}$ Data pooling by help of other publications.

${ }^{19}$ Data pooling by help of other publications.
} 
Customs Mutual Assistance Agreements (CMAAs) o

f Morocco which have entered into force ${ }^{20}$

\begin{tabular}{|c|}
\hline Country \\
\hline EU \\
\hline Turkey \\
\hline UAE \\
\hline US \\
\hline 4 CMAAs \\
\hline
\end{tabular}

Customs Mutual Assistance Agreements (CMAAs) of the Russian Federation which have entered into force ${ }^{21}$

\begin{tabular}{|c|}
\hline Country \\
\hline Canada \\
\hline EU \\
\hline India \\
\hline Japan \\
\hline Turkey \\
\hline US \\
\hline 6 CMAAs \\
\hline
\end{tabular}

Table 19

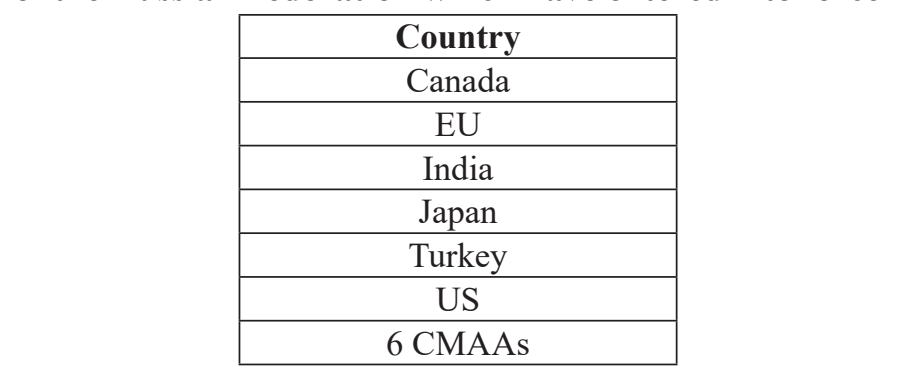

Customs Mutual Assistance Agreements (CMAAs) of Australia which have entered into force ${ }^{22}$

\begin{tabular}{|c|}
\hline Country \\
\hline Canada \\
\hline India \\
\hline Japan \\
\hline US \\
\hline 4 CMAAs \\
\hline
\end{tabular}

Table 20

Table 21

Customs Mutual Assistance Agreements (CMAAs) of New Zealand which have entered into force ${ }^{23}$

\begin{tabular}{|c|}
\hline Country \\
\hline Canada \\
\hline EU \\
\hline Japan \\
\hline Taiwan \\
\hline US \\
\hline 5 CMAAs \\
\hline
\end{tabular}

${ }^{20}$ Data pooling by help of other publications.

${ }^{21}$ Data pooling by help of other publications.

${ }^{22}$ Data pooling by help of other publications.

${ }^{23}$ Data pooling by help of other publications. 
Table 22

Customs Mutual Assistance Agreements
of South Africa which have entered int
\begin{tabular}{|c|}
\hline Country \\
\hline Canada \\
\hline EU \\
\hline Japan \\
\hline Turkey \\
\hline US \\
\hline 5 CMAAs \\
\hline
\end{tabular}

Table 23

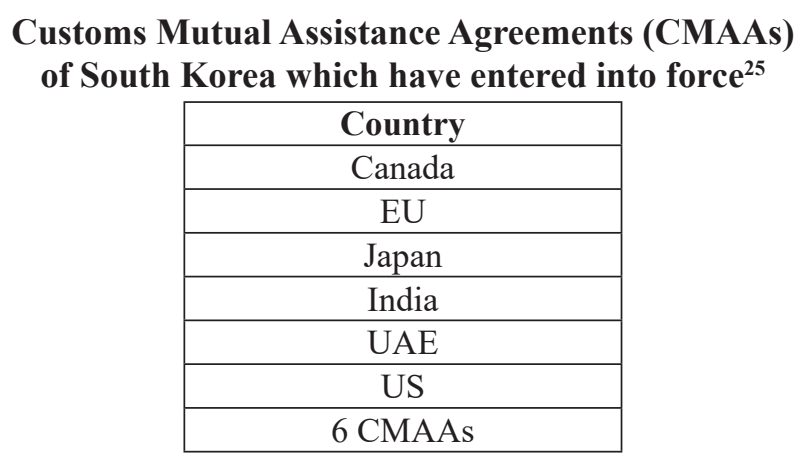

Tables 15 to 23 have been derived by help of data pooling of publications by other countries on CMAAs and they are showing that other major trade nations Australia, New Zealand, China, Russian Federation, South Africa and South Korea are also having CMAAs but also the minor trade nations Hong Kong, Algeria and Morocco.

\section{Discussion}

Data in 23 tables is showing that major global trade nations are able to negotiate CMAAs with many countries (EU: 80 bilateral agreements, US: 75 bilateral agreements, Canada: 50 bilateral agreements). However other major global trade nations have concluded only few bilateral agreements (Japan: 8 bilateral agreements, China: 7 bilateral agreements, Russian Federation: 6 bilateral agreements, South Korea: 6 bilateral agreements ${ }^{26}$ ). Medium trade nations are also able to negotiate many bilateral agreements, notably Turkey with 55 CMAAs and further nine agreements which have not entered into force.

Canada has three different approaches regarding the agreement types: CMAAs, Customs Information Sharing Memorandum of Understanding (MOU) and Customs Information Sharing Mutual Legal Assistance Treaties (MLTAs) ${ }^{27}$ (see tables 2-4).

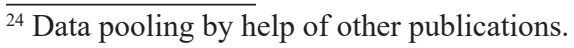

${ }^{25}$ Data pooling by help of other publications.

${ }^{26}$ Data on China and the Russian Federation have been obtained by pooling and have not been published openly; the real number of bilateral (disclosed) agreements may be higher.

27 See Canada Border Services Agency Customs Cooperation Case Study for Canada (2012), URL: https://www.wto.org/english/tratop_e/tradfa_e/case_studies_e/cc_can_e.doc, viewed on 8 April 2018.
} 
Japan has two different approaches for customs assistance agreements: CMAAs and Customs Mutual Assistance Arrangements (see tables 6, 7).

One of the big differences are the way the nations have published the agreements and data on the CMAAs - the countries in tables 1-14 have published the data on the internet whereas the CMAAs of the eight nations covered in tables 15-23 have not published any data publicly on the internet. Data pooling of other published data can give a rough picture about their activities concerning mutual customs assistance however it must be assumed that nations such as China and Russia have many more CMAAs with other countries.

\section{Conclusions}

Bilateral customs mutual assistance agreements (CMAAs) are currently the most important legal instrument of international customs co-operation since the multilateral Nairobi Convention is outdated and has never been very popular and the new Johannesburg Convention has not entered into force until $2018 .{ }^{28}$

In particular the major global trade nations US and EU have concluded many CMAAs but also medium trade nations such as Canada and Turkey have concludes numerous CMAAs (or similar types of agreements, whereas some major trade nations such as Japan have concluded only 19 CMAAs. Small trade nations have traditionally smaller negotiation power in pursing their interests but have the same obligations as their partners since the agreement are about mutual assistance and information exchange.

Many trade nations are not publishing data on their CMAAs but data pooling on other publications can share a light on their CMAAs, however such datasets stay incomplete (see tables 15-23 on China, Russia, Australia, New Zealand, South Africa, South Korea, Algeria, Morocco and Hong Kong.

The Trade Facilitation Agreement (TFA) has entered into force on 22 February 2017 has been signed by 135 WTO member states until the end of March $2018^{29}$ - these will fully implement the TFA. Many more agreements on mutual customs assistance will be negotiated, sigened and agreed.

Article 12 TFA clarifies the importance of Customs assistance and states that bilateral agreements already in force shall continue to deliver the data requested in order to combat international cross-border crime and prevent harmful attacks.

Many small trade nations should look into this issue by help of the WCO in order to make good agreements and arrangements with their neighbouring countries. The WCO bilateral model customs mutual assistance agreement is available online together with a legal commentary and advice. ${ }^{30}$

The best crime prevention can be obtained not only over long distances but with your next door countries - therefore regional customs mutual assistance is as important as inter-continental customs cooperation.

\section{References:}

1. Argentinian Customs Service, Agreements, Cooperation and Mutual Assistance, Customs Agreements, URL: http://www.afip.gob.ar/institucional/acuerdos_ingles.asp, viewed on 8 April 2018.

2. Canada Border

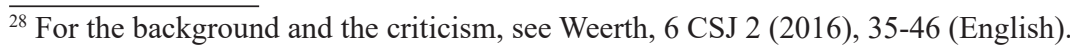

${ }^{29}$ WTO, TFAFacility.org, Ratifications as of 30 March 2018.

${ }^{30} \mathrm{WCO}, 2004$.
} 
3. Services Agency, Customs Cooperation Case Study for Canada (2012), URL: https://www.wto.org/english/tratop_e/tradfa_e/case_studies_e/cc_can_e.doc, viewed on 8 April 2018.

4. European Commission European Anti-Fraud Office, Agreements on mutual administrative assistance in customs matters, URL: http://ec.europe.eu/anti-fraud/about-us/legal-framework/customs_matters_en (2017), viewed on 8 April 2018.

5. European Commission, Press Releases on the EU-New Zealand CMAA, signed on 3 July 2017, URL: https://ec.europa.eu/taxation_customs/new-zealand_en and URL: https://ec.europa.eu/ taxation_customs/node/966_en, viewed on 8 April 2018.

6. Government of India Department of Revenue, International Cooperation, URL: http://www.dor.dov.in/international_cooperation_pml, viewed on 8 April 2018.

7. Japan Customs, Customs Mutual Assistance Agreement (CMAA), URL: http://www.customs.go.jp/english/cmaa/index.htm, viewed on 8 April 2018.

8. Mauritian Customs Service, Legislation, Customs, URL: http://www.mra.mu/index.php/ legislations, viewed on 8 April 2018.

9. Republic of China (Taiwan)

10. Ministry of Finance, Customs Mutual Assistance Agreement (CMAA), URL: http://www.mof.gov.tw/Eng/Pages/Detail.aspx?nodeid=264\&pid=64538, viewed on 8 April 2018.

11. Stankevicius, R., (2007) Problems of International Cooperation and Mutual Assistance Among the European Union Customs Authorities, 2 Intellectual Economics 2, pp. 60-67.

12. Turkish Customs Service, The Agreement on Co-Operation and Mutual Assistance in Customs Matters, URL: https://english.gtb.gov.tr/external-relations/bilateral-relations/the-agreement-oncooperation-and-mutual-assistance-in-customs-matters, viewed on 8 April 2018.

13. UAE Customs Service, Agreements between UAE and Other Countries, URL: https://www.fca.gov.ae/en/homerightmenu/pages/bilateralagreements.aspx?SelectedTab=7, viewed on 8 April 2018.

14. UNECE, Trade Facilitation Implementation Guide, Customs Cooperation, URL: http://tfig. unece.org/contents/custom-cooperation.htm, viewed on 8 April 2018.

15. US Customs and Border Protection, Customs Mutual Assistance Agreements (CMAA), URL: https:/www.cbp.gov/border-security/international-initiatives/international-agreements/cmaa, viewed on 8 April 2018.

16. US Customs and Border

17. Protection, Customs Mutual Assistance Agreements (CMAA), Overview dated January 2014, published by the WCO, URL: http://www.wcoomd.org/media/wco/public/global/pdf/topics/wto-atf/ dev/cbps-cmaa.pdf, viewed on 8 April 2018.

18. WCO, Revised Model Bilateral Agreement on Mutual Customs Assistance in Customs Matters, Introduction, Text and Commentary, URL: http://www.wcoomd.org/-/media/wco/public/global/ pdf/topics/enforcement-and-compliance/tools-and-instruments/model-agreement.pdf?la=en, June 2004, viewed on 8 April 2018 (WCO, 2004).

19. WCO, (2012) Benefits of the Johannesburg Convention, URL: http://www.wcoomd.org/ layouts/ConstructionKit/SolrLinkHandler.ashx/? id=99AE6B2CF41A4A0482EEB677F2C8CCOE\&lang=en, viewed on 8 April 2018.

20. WCO, About Us aWCO, Mutual Administrative Assistance Instruments, URL: http://www. wcoomd.org/en/topics/enforcement-and-compliance/instruments-and-tools/wco-and-international-instruments-on-mutual-administrative-assistance.aspx, viewed on 8 April 2018 (WCO, 2018b).

21. Weerth, C. The Johannesburg Convention on Mutual Customs Assistance, 6 Customs Scientific Journal 2 (2016), pp. 35-46.

22. WTO, List of WTO-Members and WTO-Observers, URL: https://www.wto.org/english/ thewto_e/whatis_e/tif_e/org6_e.htm, viewed on 8 April 2018 (WTO, 2018a).

23. WTO, The Trade Facilitation Facility, Ratifications and Notifications, URL: http://www.tfafacility.org/ratifications, viewed on 8 April 2018 (WTO, 2018b). 


\section{УГОДИ ПРО ВЗАЄМНУ ДОПОМОГУ В МИТНИХ СПРАВАХ В КОНТЕКСТІ СТАТТІ 12 УГОДИ ПРО СПРОЩЕННЯ ПРОЦЕДУР ТОРГІВЛІ - СВІТОВА ПЕРСПЕКТИВА}

\section{Карстен BIPC,}

Головне митне управління міста Бремен, Федеральна митна служба Німеччини, Університет прикладних наук в галузі економіки та менеджменту, Бремен, Німеччина carsten.weerth@gmx.de

Митна співпрачя на міжнародному, регіональному та світовому рівнях спрямована на забезпечення дотримання чинного законодавства та нормативних актів, а також на поліпшення контролю за торговельним потоком шляхом обміну інформацією про такі митні особливості як транзит, декларація експорту та імпорту, походження, иінність товару та відомості про торговия, зокрема інформація про митне шахрайство. Цей обмін даними в даний час є ключовим елементом Угоди СОТ про спрощення процедур торгівлі, а стаття 12 Угоди розглядає всі необхідні компоненти для обміну інформачією, такі як потреба запитуючої краӥни, перевірка запиту, захист обмінюваних даних та забезпечення конфіденційність даних та обмін даними на основі принщипу взаємності. У Статті 12 Угоди СОТ про спрощення прочедур торгівлі також йдеться про двосторонні та регіональні угоди про взаємну адміністративну допомогу, які залишаються основним інструментом урядів та митних адміністращій для участі в такій співпраці. Автор вивчає різні підходи та представляє список Угод про взаємну митну допомогу в митних справах зацікавлених сторін у міжнародній торгівлі, країн з середнім рівнем розвитку торгівлі та малих краӥн. 23 таблиці відображають результати иьього дослідження. Стаття досліджує Угоди про взаємну митну допомогу, використовуючи публікаиії, які напряму стосуються питання Угод про взаємну митну допомогу та інших краӥн, які не публікують дані про свої Угоди ВМД шляхом об'єднання даних, опублікованих іншими країнами. Автор робить висновок, щзо двосторонні взаємні угоди є сприятливими для великих країн, але викликають труднощі в частині переговорів для малих краӥн. Як результат, в статті обтрунтовується важливість митного співробітництва на двосторонній регіональній основі.

Ключові слова: митне право, митна класифікація, тарифні ставки, номенклатура Гармонізованої системи (ГС), загальні правила, товарна позиція, примітки, примітки до субпозицій, додаткові примітки, Єдиний митний тариф (СМТ), Комбінована номенклатура (КН), Європейський Союз (СС). 